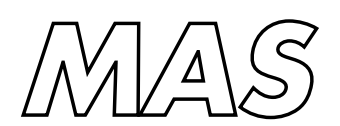

Modelling, Analysis and Simulation Modelling, Analysis and Simulation
MAS A fully conservative model for compressible two-fluid
flow

J. Wackers, B. Koren

Report MAS-e0534 December 2005 
$\mathrm{CWI}$ is the National Research Institute for Mathematics and Computer Science. It is sponsored by the Netherlands Organization for Scientific Research (NWO).

$\mathrm{CWI}$ is a founding member of ERCIM, the European Research Consortium for Informatics and Mathematics.

CWI's research has a theme-oriented structure and is grouped into four clusters. Listed below are the names of the clusters and in parentheses their acronyms.

Probability, Networks and Algorithms (PNA)

Software Engineering (SEN)

\section{Modelling, Analysis and Simulation (MAS)}

Information Systems (INS)

Copyright (C) 2005, Stichting Centrum voor Wiskunde en Informatica

P.O. Box 94079, 1090 GB Amsterdam (NL)

Kruislaan 413, 1098 SJ Amsterdam (NL)

Telephone +31205929333

Telefax +31205924199

ISSN 1386-3703 


\title{
A fully conservative model for compressible two-fluid flow
}

\begin{abstract}
A five-equation model for compressible two-fluid flow is proposed, that is based on physical flow equations only. The model is conservative and pressure-oscillation free. Equations for continuous flow and jump conditions for discontinuities are given, as well as a discretization of the equations and an adaptation of the HLL Riemann solver to two-fluid flow. Numerical tests in $1 \mathrm{D}$ and $2 \mathrm{D}$ show the accuracy of the method.
\end{abstract}

2000 Mathematics Subject Classification: 35L65, 35L67, 65N30, 76N15, 76T99

Keywords and Phrases: compressible two-fluid flow; interface capturing; conservation Note: This research has been funded by the Dutch BSIK/BRICKS project. 



\title{
A fully conservative model for compressible two-fluid flow
}

\author{
Jeroen Wackers ${ }^{*} \dagger$ and Barry Koren ${ }^{\ddagger}$ \\ CWI, P.O. Box 94079, 1090 GB Amsterdam, The Netherlands
}

\begin{abstract}
SUMMARY
A five-equation model for compressible two-fluid flow is proposed, that is based on physical flow equations only. The model is conservative and pressure-oscillation free. Equations for continuous flow and jump conditions for discontinuities are given, as well as a discretization of the equations and an adaptation of the HLL Riemann solver to two-fluid flow. Numerical tests in 1D and 2D show the accuracy of the method. Copyright (c) 2005 John Wiley \& Sons, Ltd.
\end{abstract}

KEY WORDS: compressible two-fluid flow; interface capturing; conservation

\section{INTRODUCTION}

In compressible two-fluid flows, the flow medium consists of two fluids that do not mix, separated by a sharp interface. Interface-capturing methods for these flows model this interface as a mixture region, a numerically smeared transition from fluid 1 to fluid 2. Abgrall and Karni [1] have shown that many conservative formulations of such models produce large pressure errors. This problem can be solved by using locally non-conservative methods [1] or by solving the full two-phase flow model [2].

Here, an intermediate approach is presented. The current method is fully conservative and pressure-oscillation free, but it is simpler than two-phase methods, because it has a single pressure and velocity for the two fluids. It is an extension of the work by Van Brummelen and Koren [3], it is described in detail in Reference [4]. Flow equations are derived from a physics-based model which allows an exact closure for flow discontinuities; an improvement over similar methods, like the method by Kapila et al. [5].

The present method has two major advantages. First, the conservative formulation gives good capturing of shocks and interfaces, also for problems with very strong shocks. And second, the model strongly resembles a single-fluid model: it does not require a complex

\footnotetext{
*Correspondence to: Jeroen Wackers, CWI, P.O. Box 94079, 1090 GB Amsterdam, The Netherlands.

†E-mail: Jeroen.Wackers@cwi.nl

‡E-mail: Barry.Koren@cwi.nl
} 
interface-tracking algorithm. It can thus be solved with existing techniques, even on complex, irregular grids.

\section{FLOW MODEL}

The numerical model used here for the two-fluid flow is based on a physical mixture model. The fluids, however, are not fully mixed: the 'mixture' may be thought to consist of very small bits of the two pure fluids, in an arbitrary pattern. Each fluid has its own pure-fluid equation of state and the fluids interact only by exerting forces on each other. In the model, the pressure and the velocity of the fluids are equal, but each fluid has its own density. The volume fraction of fluid $1, \alpha$, is used to denote the relative amounts of the two fluids. Thus, in $1 \mathrm{D}$, we have five independent state variables $\left(p, u, \rho_{1}, \rho_{2}\right.$ and $\left.\alpha\right)$, so we need five differential equations to solve the flow.

The bulk two-fluid flow satisfies the standard Euler equations

$$
\begin{aligned}
(\rho)_{t}+(\rho u)_{x} & =0 \\
(\rho u)_{t}+\left(\rho u^{2}+p\right)_{x} & =0 \\
(\rho E)_{t}+(\rho E u+p u)_{x} & =0
\end{aligned}
$$

In these equations, the bulk density $\rho$ and bulk total energy $E$ are

$$
\rho=\alpha \rho_{1}+(1-\alpha) \rho_{2}, \quad \rho E=\alpha \rho_{1} E_{1}+(1-\alpha) \rho_{2} E_{2}
$$

with the total energy for each fluid $j=1,2$ defined as $E_{j}=e_{j}+\frac{1}{2} u^{2}$. Here $e_{j}$ is the internal energy of fluid $j$.

Two more flow equations are needed to close the system. The first one is the conservation of mass for fluid 1: the fluids are not supposed to change into each other. Using the partial density $\rho_{1} \alpha$, this equation is

$$
\left(\rho_{1} \alpha\right)_{t}+\left(\rho_{1} u \alpha\right)_{x}=0
$$

Together with Equation (1a), this equation gives mass conservation for both fluids. For the last equation, the energy balance of fluid 1 is used. This equation has a special property: the fluids exert forces on each other, so they exchange energy. This exchange appears as a source term in the equation:

$$
\left(\rho_{1} E_{1} \alpha\right)_{t}+\left(\rho_{1} E_{1} u \alpha+p u \alpha\right)_{x}=S
$$

An expression for this source term is derived in the next section.

To close the system, equations of state (EOS) are needed for the two fluids. A possible EOS is the ideal gas law

$$
p=\left(\gamma_{1}-1\right) \rho_{1} e_{1}=\left(\gamma_{2}-1\right) \rho_{2} e_{2}
$$

with constant $\gamma$ 's. For this equation, it is easy to compute the primitive variables $p$ and $\alpha$ from the total energies. 


\section{THE SOURCE TERM}

\subsection{Derivation of the source term}

The source term $S$ in Equation (3b) models the exchange of energy between fluids 2 and 1 . Euler flow has no heat conduction, so the only energy exchanged is the work done by the force between the fluids. This force is found from a momentum analysis.

Consider a fluid element in a smooth 1D flow (Figure 1). The element contains fluids 1 and 2 (the interface is drawn schematically). The force on the entire fluid element is $p(x)-p(x+\mathrm{d} x)$ and its bulk mass is $\rho \mathrm{d} x$. The force on fluid 1 in the element is $(p \alpha)(x)-$ $(p \alpha)(x+\mathrm{d} x)+S_{M} \mathrm{~d} x$. Its mass is $\rho_{1} \alpha \mathrm{d} x$ and its acceleration is equal to the acceleration of the entire element (because both fluids have the same velocity). Therefore,

$$
\frac{p(x)-p(x+\mathrm{d} x)}{\rho \mathrm{d} x}=\frac{(p \alpha)(x)-(p \alpha)(x+\mathrm{d} x)+S_{M} \mathrm{~d} x}{\rho_{1} \alpha \mathrm{d} x}
$$

The force $S_{M}$ follows from this expression (using the mass fraction $\beta=\rho_{1} \alpha / \rho$ ):

$$
S_{M}=p \alpha_{x}+(\alpha-\beta) p_{x}
$$

The first term, $p \alpha_{x}$, expresses the pressure force on the interface: pressure times the projected height of the interface. The second term is friction. There is no friction in normal Euler flow, but the two fluids are mixed so well that friction force between the fluids prevents one fluid from moving faster than the other. When fluid 1 is denser than fluid $2(\beta>\alpha)$, then it is accelerated less by the pressure force $-p_{x}$ than the lighter fluid 2 . In that case, the friction force passes a part of the pressure force on fluid 2 to fluid 1, such that the velocity of the two fluids remains the same. The energy source term $S$ is the work done by the force $S_{M}$

$$
S=u S_{M}=p u \alpha_{x}+(\alpha-\beta) u p_{x}
$$

\subsection{Characteristic analysis of the system for ideal gas}

The source term (6) is valid for any EOS. Substitution of the ideal gas law (4) allows a characteristic analysis of the flow equations. This results in five wave speeds

$$
\lambda_{1}=u-c, \quad \lambda_{2,3,4}=u, \quad \lambda_{5}=u+c, \quad \text { with } c=\sqrt{\left(\gamma_{1} \alpha+\gamma_{2}(1-\alpha)\right) p / \rho}
$$

This combination of wave speeds is physically correct. It can be proved that (6) is the only possible source term that gives such a combination.

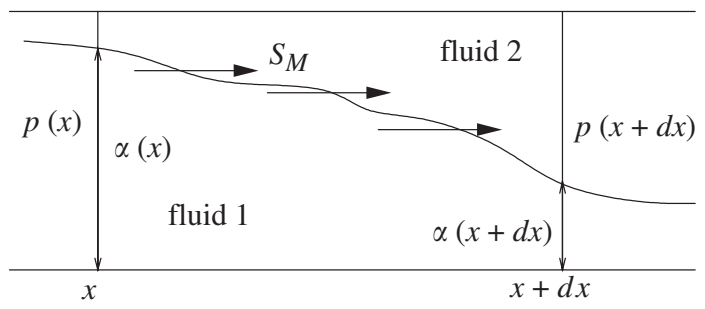

Figure 1. Two-fluid element in smooth 1D flow. 


\subsection{Source term in discontinuities}

To allow weak solutions with discontinuities of the two-fluid flow equations, we need a proper definition of the flow across a discontinuity. The first four equations, (1a)-(1c) and (3a), satisfy the standard Rankine-Hugoniot jump condition $\Delta \mathbf{f}=c_{s} \Delta \mathbf{q}$, with $c_{s}$ the speed of the discontinuity. For the fifth equation, $(3 \mathrm{~b})$, this condition becomes

$$
\Delta \mathbf{f}=c_{s} \Delta \mathbf{q}+\int_{x_{\mathrm{L}}}^{x_{\mathrm{R}}} S \mathrm{~d} x
$$

The integral must be evaluated across the discontinuity, which is impossible. However, if we assume that the discontinuity is the limit of a viscous discontinuity and thus has a continuous internal structure (the precise shape is unimportant), then we can write the state variables as continuous functions of $p$ and integrate the source term

$$
\int_{x_{\mathrm{L}}}^{x_{\mathrm{R}}} S \mathrm{~d} x=\Delta(p u \alpha)+\frac{1}{2} \beta_{\mathrm{L}} \rho_{\mathrm{L}}\left(u_{\mathrm{L}}-c_{s}\right) \Delta u^{2}+\frac{1}{\rho_{\mathrm{L}}\left(u_{\mathrm{L}}-c_{s}\right)} \int_{p_{\mathrm{L}}}^{p_{\mathrm{R}}} p \alpha \mathrm{d} p
$$

A derivation of this expression is given in Reference [4]. The last integral can be evaluated by integration of the fifth equation, (3b), combined with an EOS. So there is a unique jump condition for the present two-fluid model, but, unlike the single-fluid jump condition, it depends on the material properties of the fluids.

\section{NUMERICAL METHOD}

\subsection{Second-order accurate discretization}

The flow equations are discretized with a second-order accurate finite-volume scheme. Fluxes are computed with an improved version of Linde's three-wave HLL approximate Riemann solver [6], combined with a limited reconstruction of the cell interface states. The limiter is applied to the primitive variables $\rho, u, p, \alpha$ and $\beta$. Time stepping is done with a two-step scheme [7]

$$
\begin{aligned}
& \mathbf{q}_{i}^{k+1}=\mathbf{q}_{i}^{k}-\frac{\Delta t}{\Delta x}\left(\mathbf{f}_{i+1 / 2}^{k}-\mathbf{f}_{i-1 / 2}^{k}\right)+\frac{\Delta t}{\Delta x} \mathbf{s}_{i}^{k} \\
& \mathbf{q}_{i}^{k+2}=\mathbf{q}_{i}^{k}-2 \frac{\Delta t}{\Delta x}\left(\mathbf{f}_{i+1 / 2}^{k+1}-\mathbf{f}_{i-1 / 2}^{k+1}\right)+2 \frac{\Delta t}{\Delta x} \mathbf{s}_{i}^{k+1}
\end{aligned}
$$

\subsection{Numerical source term}

A discretization of the source term is needed in two places. First, an approximation of the source term in a discontinuity is needed to compute the HLL flux. The HLL solver models a Riemann problem with three discontinuous waves. The easiest way to incorporate the source term in these waves is to compute only one approximate solution of Equation (9), using the left and right cell interface state, and to divide this source term proportionally over the three waves. This procedure causes some small inaccuracies, but it is fast and straightforward.

Secondly, the source term for the time integration, $\mathbf{s}_{i}$ in Equation (10), is computed. It consists of two parts: (i) Sources in the discontinuities at the cell faces. These are summed 
over all HLL waves, on interfaces $i-\frac{1}{2}$ and $i+\frac{1}{2}$, that actually run into cell $i$. (ii) Sources in the continuous flow in the cell. These are integrated over the piecewise linear approximations to the primitive variables, that follow from the use of the limiter.

\section{NUMERICAL RESULTS}

\subsection{Shock tube tests}

The method is tested first on a 1D Riemann problem for ideal gases. The results are compared with the exact solutions. The test is a two-fluid variation of Sod's problem, with a ten times higher left pressure and density, giving it a pressure ratio of 1:100. Figure 2 shows that the discontinuities (shock and two-fluid interface) are in the proper locations. The pressure is constant over the contact discontinuity and the volume fraction is constant over the shock and over the expansion fan: no pressure oscillations occur. A convergence study for this particular problem, on five grids, shows that the $L^{1}$-errors in $\rho, u$ and $p$ converge approximately with the power 0.96 of the mesh width. The volume fraction converges with the power 0.78 of the mesh width. This rate of convergence is comparable to that for single-fluid solutions with limited second-order schemes.

\subsection{Shock hitting helium bubble}

This 2D test case has been taken from literature [8,9]. It consists of a cylindrical helium bubble in air, which is hit by an incoming shock wave. The problem is solved on a grid of $200 \times 400$ cells, with $\Delta t=1.25 \times 10^{-5}$. Figure 3 shows the solution at two times. The (half) bubble is visible between $x=-0.025$ and 0.025 . The incident shock, coming from the right, can be seen in the air above the bubble, the curved shock in the bubble runs ahead of this shock. The rightmost wave is an expansion wave, reflected into the air behind the shock. At the later time, a complicated $\lambda$-shock structure has developed above the bubble. Figure 4 shows the pressure and the volume fraction for this time. Of the waves appearing in the density plot, the shock waves and expansions are visible in the pressure plot only and the interface in the volume fraction plot only, as it should be. The pressure is continuous over the interface.
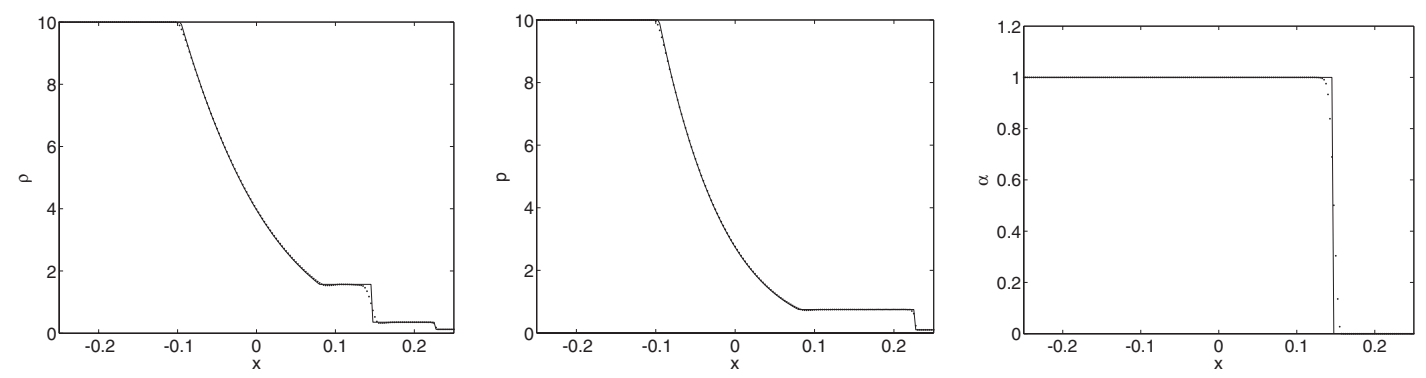

Figure 2. High-pressure, two-fluid Sod problem. $(\rho, u, p)_{\mathrm{L}}=(10,0,10)$, $(\rho, u, p)_{\mathrm{R}}=(0.125,0,0.1), \gamma_{\mathrm{L}}=1.4$ and $\gamma_{\mathrm{R}}=1.6$. The grid has 200 cells, 160 time steps, $\Delta t / \Delta x=0.2(\mathrm{CFL}=0.56)$. Solid lines: exact solution. 

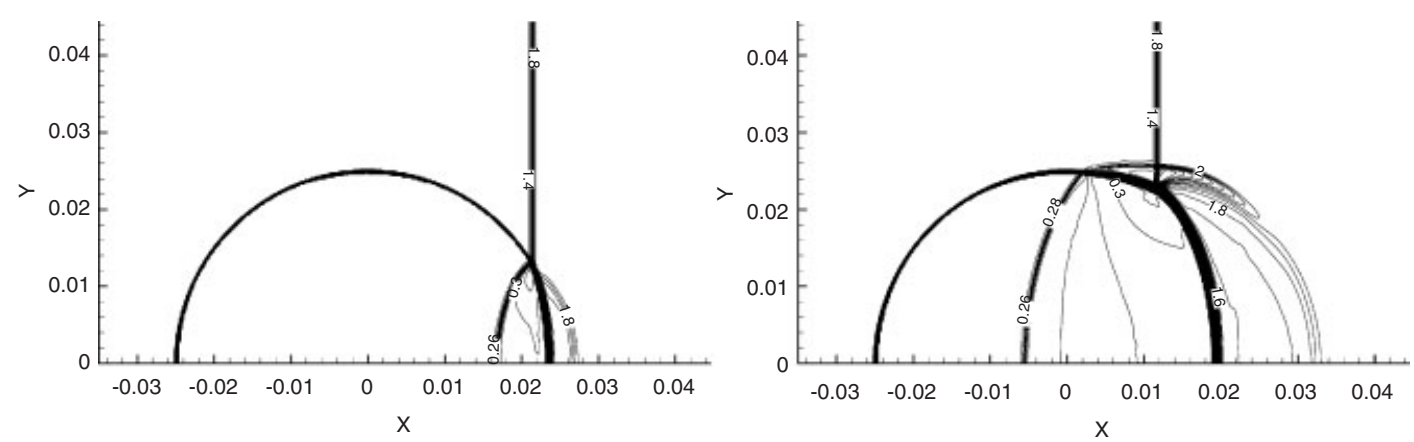

Figure 3. Shock hitting helium bubble, density at $t=2.74 \times 10^{-3}$ and $t=10.74 \times 10^{-3}$.
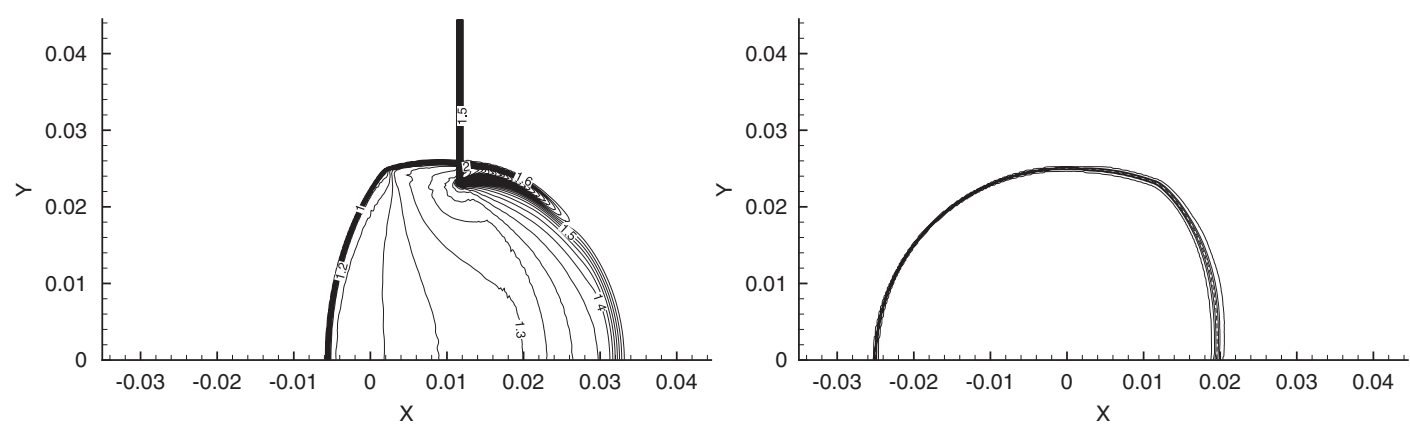

Figure 4. Shock hitting helium bubble, pressure (left) and volume fraction (right) at $t=10.74 \times 10^{-3}$.

The speeds of the shocks and the interface at the centerline $(y=0)$ have been compared with results from Quirk and Karni [9] (obtained on a very fine, adapted grid). The difference is between 0.7 and $2.2 \%$.

\section{CONCLUSION}

A model for compressible two-fluid flow is proposed, that is conservative and pressureoscillation free. 1D tests show that the model resolves contact discontinuities without creating pressure errors and that it accurately handles problems with strong shocks. A $2 \mathrm{D}$ test confirms that the method also handles curved shocks and interfaces well.

\section{REFERENCES}

1. Abgrall R, Karni S. Computations of compressible multifluids. Journal of Computational Physics 2001; 169:594-623.

2. Abgrall R, Saurel R. Discrete equations for physical and numerical compressible multiphase mixtures. Journal of Computational Physics 2003; 186:361-396. 
3. Van Brummelen EH, Koren B. A pressure-invariant conservative Godunov-type method for barotropic two-fluid flows. Journal of Computational Physics 2003; 185:289-308.

4. Wackers J, Koren B. Five-equation model for compressible two-fluid flow. Technical Report MAS-E0414, CWI: Amsterdam, http://ftp.cwi.nl/CWIreports/MAS/MAS-E0414.pdf, 2004.

5. Kapila AK, Bdzil JB, Menikoff R, Son SF, Steward DS. Two-phase modelling of DDT in granular materials: reduced equations. Physics of Fluids 2001; 13:3002-3024.

6. Linde T. A practical, general-purpose, two-state HLL Riemann solver for hyperbolic conservation laws. International Journal for Numerical Methods in Fluids 2002; 40:391-402.

7. Wackers J, Koren B. A simple and efficient space-time adaptive grid technique for unsteady compressible flows. AIAA Paper 2003-3825. American Institute of Aeronautics and Astronautics, 2003.

8. Haas J-F, Sturtevant B. Interaction of weak shock waves with cylindrical and spherical gas inhomogeneities. Journal of Fluid Mechanics 1987; 81:41-76.

9. Quirk JJ, Karni S. On the dynamics of a shock-bubble interaction. ICASE Report 94-75. NASA Langley Research Center: Hampton, VA, 1994. 